\title{
Internists' Perceptions of Proton Pump Inhibitor Adverse Effects and Impact on Prescribing Practices: Results of a Nationwide Survey
}

\author{
Jacob E. Kurlander a, b, c, h, Mark Kolbe ${ }^{\mathrm{a}}$, Joel H. Rubenstein ${ }^{\mathrm{a}, \mathrm{c}, \mathrm{d}}$, Caroline R. Richardson ${ }^{\mathrm{c}, ~ e}$, \\ Michele Heisler ${ }^{\mathrm{a}, \mathrm{c}, \mathrm{d}}$, Arlene Weissman ${ }^{\mathrm{f}}$, Raymond De Vries ${ }^{\mathrm{g}}$, Sameer D. Saini ${ }^{\mathrm{a}, \mathrm{c}, \mathrm{d}}$
}

\begin{abstract}
Background: Observational studies have linked proton pump inhibitors (PPIs) with serious adverse effects. The study aimed to evaluate internists' perceptions of PPI harms and effects on prescribing.

Methods: This was an online survey of a representative sample of the American College of Physicians in 2013. We queried familiarity with and concern about PPI adverse effects (1 - 7 Likert-type scales, anchored by "not at all" and "extremely"). We also asked how frequently (often, sometimes, rarely, or never) participants used any of three "de-escalation" strategies to stop or reduce PPIs because of concern about adverse effects: reducing patients' PPI dose, switching to $\mathrm{H} 2$ blocker, or discontinuing PPI. We used multivariable logistic regression to evaluate associations between sometimes/often using any PPI de-escalation strategy and gender, time in practice, familiarity, and concern.
\end{abstract}

Results: The response rate was 53\% (487/914). Seventy percent were male, median time in practice was $11-15$ years, and most practiced general medicine (58\%). Ninety-nine percent reported at least some familiarity with reported adverse effects (mean 4.9, standard deviation (SD) 1.0), and 98\% reported at least some concern (mean 4.6, SD 1.3). Sixty-three percent reported sometimes/often reducing the PPI dose, 52\% switching to H2 blocker, and 44\% discontinuing PPI.

Manuscript submitted December 11, 2017, accepted December 27, 2017

${ }^{a}$ Department of Internal Medicine, University of Michigan, Ann Arbor, MI, USA

${ }^{b}$ Veterans Affairs Ann Arbor Health Care System, Ann Arbor, MI, USA

'Institute for Healthcare Policy and Innovation, University of Michigan, Ann Arbor, MI, USA

${ }^{\mathrm{d} V A}$ Ann Arbor Center for Clinical Management Research, Ann Arbor, MI, USA

eDepartment of Family Medicine, University of Michigan, Ann Arbor, MI, USA

fResearch Center, American College of Physicians, Philadelphia, PA, USA ${ }^{g}$ Center for Bioethics and Social Sciences in Medicine, University of Michigan, Ann Arbor, MI, USA

${ }^{\mathrm{h}}$ Corresponding Author: Jacob Kurlander, Department of Internal Medicine, University of Michigan, 3912 Taubman Center, 1500 E. Medical Center Dr., SPC 5362, Ann Arbor, MI 48109-5362, USA. Email: jkurland@umich.edu

doi: https://doi.org/10.14740/gr944w
In multivariable analysis, familiarity with adverse effects (OR 1.66 (1.31 - 2.10) for 1-point increase, $\mathrm{P}<0.001)$ and concern (OR 2.14 $(1.76$ - 2.61) for 1 -point increase, $\mathrm{P}<0.001)$ were independently associated with de-escalation. Gender and time in practice had no effects.

Conclusion: Almost all internists report awareness and concern about PPI adverse effects, and most are de-escalating PPIs as a result. Research on which approach is most effective for which patients is critically important.

Keywords: Side effect; Risk; Provider

\section{Introduction}

Proton pump inhibitors (PPIs) are among the most widely used drug classes in the United States [1]. However, with their increasing use, there are come concerns about misuse and overuse [2]. Physicians often prescribe PPIs without a clear indication, at higher doses, and for a longer duration than necessary [3]. Inappropriate PPI use is now attracting increasing scrutiny because of concerns about a range of possible PPI adverse effects. In epidemiologic studies, PPIs have been associated with pathology in almost every organ system [4], including stroke [5], dementia [6], pneumonia [7], gastric cancer [8], bone fracture [9], Clostridium difficile infection [10], kidney disease [11], and cardiac events [12]. However, the magnitude of these associations tends to be small, contributing to debate about the nature of these associations, which may mainly be explained by confounding variables rather than causation $[13,14]$.

In the face of this growing body of research, little is known about how prescribers perceive or react to possible but uncertain adverse effects from PPIs. On the one hand, it is well known that changes in medical evidence often take more than a decade to change clinical practice $[15,16]$, and this inertia might also attenuate the response of physicians to reports of possible adverse effects. On the other hand, lay media have widely covered studies of adverse effects $[17,18]$, and patients are likely to discuss the topic with their physicians. If providers are changing their prescribing habits, another important question is how? Multiple strategies may be used to de-escalate (i.e., stop or reduce) PPIs, including abrupt PPI withdraw- 
al [19], step down therapy, and $\mathrm{H} 2$ blocker substitution [20]. Whether, and how, to de-escalate PPI in an individual patient requires careful consideration of the patient and the PPI indication. We have shown that physicians tend to poorly discriminate between patients who are likely to benefit from long-term PPI use and those who are not [21]. Thus, switching PPI to an $\mathrm{H} 2$ blocker would provide inferior gastroprotection in patients at high risk for peptic ulcer disease, for example [22].

In order to understand more about how data on PPI adverse effects have affected real-world practice, we conducted a nationwide survey of internists in the United States to investigate perceptions and attitudes regarding PPI adverse effects and the effects of these on PPI prescribing. We hypothesized that a high proportion of physicians are taking steps to de-escalate PPIs, and that physicians with greater familiarity with and concern about PPI adverse effects would be more likely to do so.

\section{Materials and Methods}

In collaboration with the American College of Physicians (ACP), we conducted a web-based national survey of physicians, including trainees and independent practitioners in general practice or any medical subspecialty.

\section{Study population}

Our participants were members of the Internal Medicine Insider Research panel maintained by the ACP Research Center [23]. Excluding medical students, affiliate members (non-physicians), honorary fellows, and non-US members, the panel is a representative group of 1,000 ACP members who have volunteered to participate in periodic physician surveys in return for points redeemable for gift cards. The panel was started in 2011 and is regularly adjusted to represent ACP membership across multiple demographics. Within this panel, we excluded clinicians who self-reported less than $25 \%$ of their time in direct patient care. We identified 914 physicians who met these pre-established criteria for survey administration.

\section{Survey distribution}

The first email invitation was sent to the 914 physicians by email on November 11, 2013. Three additional reminder emails were sent to non-responders during the survey period of 10 days. Each participant was given $\$ 10$ for completing the survey.

\section{Questionnaire development and content}

We developed a 29-item multiple-choice survey that was piloted among 10 gastroenterology and internal medicine attending physicians prior to distribution. Feedback was used to iteratively modify the survey instrument. The survey con- tained questions about physicians' familiarity with published evidence on the adverse effects of PPIs, level of concern about adverse effects, the extent to which "recent studies about longterm harms of PPI use changed your PPI prescribing practices", and perception of the likelihood that "long-term use of a PPI increases" any of three specific adverse effects (bone loss, bowel infections, and pneumonia).

For all of these questions, response options were on a seven-point Likert-type scale anchored by "not at all" and "extremely". The survey focused on these three adverse effects because they had been most widely discussed in the medical literature at the time of the survey. We also asked about awareness of the three possible adverse effects (yes/no), which adverse effect is most clinically important, and how frequently participants either reduced patients' PPI dose, switched a PPI to an $\mathrm{H} 2$ blocker, or simply stopped a PPI because of concerns about PPI adverse effects (often, sometimes, rarely or never).

Respondents provided information on their age (by decade of life), gender, race, trainee status (resident or fellow), specialization, board certification, time in practice (in 5-year intervals), clinical volume, and academic affiliation. Practice characteristics were not obtained for residents or fellows.

\section{Analysis}

For categorical variables, we calculated descriptive statistics, including frequency counts and percentages. For questions that used the seven-point Likert-type scale, we summarized scores with the mean and standard deviation (SD), as well as reported the fraction of respondents with scores greater than 1 (not at all). For analysis of how respondents have changed their practices in response to concerns about PPI adverse effects, responses were dichotomized as rarely/never vs. sometimes/ often, as others have done [24, 25]. Chi-square tests were used to compare respondents' frequency of using the three different strategies to minimize PPI use (dose reduction, switching to an H2 blocker, or simply stopping PPI), and to compare rates of awareness of the three adverse effects. To compare the perceived likelihood that PPIs increase the risk of the three adverse effects, linear regression was used with likelihood of adverse effect (on a $1-7$ scale) as the dependent variable and the specific adverse effect as the sole independent indicator variable.

To determine correlates of using PPI de-escalation strategies, we first evaluated bivariate associations between sometimes/often using any of the three PPI de-escalation strategies and four pre-specified variables: gender, years in practice, and familiarity with and concern about PPI adverse effects. We then performed multivariable logistic regression using the same four predictor variables. Concern about PPI adverse effects and familiarity with published data on PPI adverse effects were analyzed as continuous variables from 1 to 7 .

For years in practice, trainees were assigned 0 . Age was not analyzed because of collinearity with years in practice. Predicted probabilities of PPI de-escalation were calculated for selected levels of concern about PPI harms to illustrate regression results in the form of absolute probabilities. All reported analyses are for two-tailed testing with $\mathrm{P}<0.05$ con- 
sidered statistically significant. Stata version 14 was used for all analyses.

This study was deemed exempt from IRB review at the University of Michigan.

\section{Results}

\section{Respondent characteristics}

Of the 914 invited participants, 487 completed the survey for a response rate of $53 \%$. A majority of respondents were male $(70 \%)$, and the median age was 40 - 49 years old (Table 1). Twenty-two percent were trainees, $58 \%$ practiced general medicine, $2 \%$ gastroenterology, and $18 \%$ another internal medicine subspecialty. Among attending physicians, 97\% were board certified, the median time in practice was $11-15$ years, $49 \%$ had an academic affiliation, and most (64\%) saw at least 50 patients in a typical work week.

\section{Knowledge and perceptions of PPI harms}

On a 1 - 7 scale anchored by "not at all" and "extremely", $99 \%$ of respondents reported at least some familiarity (score $>$ 1) with published scientific data on possible PPI harms (mean 4.9, SD 1.0), and 98\% of respondents reported at least some concern (score $>1$ ) about long-term harms when prescribing PPIs (mean 4.6, SD 1.3). Ninety-two percent of respondents reported that they had made at least some change (score $>1$ ) in their prescribing practices (mean 4.2, SD 1.5). These results are shown in Figure 1.

Eighty-five percent of respondents were aware of bone loss as a possible PPI adverse effect, 73\% were aware of pneumonia, and 68\% were aware of bowel infection $(\mathrm{P}<0.001)$. The largest proportion of respondents (44\%) were aware of all three possible adverse effects, while only $2 \%$ were aware of none. Most respondents $(51 \%)$ believed that bone loss was the most clinically important adverse effect, followed by bowel infection (28\%) and pneumonia (21\%).

On the 1 - 7 scale anchored by "not at all likely" and "extremely likely", the mean levels of perceived likelihood that PPI increases the risk of bone loss, bowel infection, and pneumonia were 4.7 (SD 1.1), 4.5 (SD 1.2), and 4.4 (SD 1.2), respectively $(\mathrm{P}<0.001$ for bowel infection and pneumonia vs. bone loss) (Fig. 2).

\section{Changes in PPI prescribing behavior}

Twenty-three percent "often" and 50\% "sometimes" made changes in PPI prescriptions because of concerns about adverse effects. Respondents used a range of different strategies for de-escalating PPIs. Reducing PPI dose was the most frequently used approach (62.6\%), followed by switching to an $\mathrm{H} 2$ blocker (51.5\%), and discontinuing PPI (43.9\%; $\mathrm{P}<0.001)$. In bivariate analyses, both familiarity with PPI harms (OR 1.87 (1.51 - 2.31) for 1-point increase on seven-point scale, $\mathrm{P}$
$<0.001)$ and concern about PPI harms (OR 2.26 (1.87 - 2.74) for 1-point increase on seven-point scale, $\mathrm{P}<0.001)$ were associated with sometimes/often de-escalating PPIs. Gender and years in practice did not have significant effects.

In multivariable regression analysis, familiarity with published data on possible harms (OR 1.66 (1.31 - 2.10) for 1 -point increase on seven-point scale, $\mathrm{P}<0.001)$ and concern about PPI adverse effects (OR 2.14 (1.76 - 2.61) for a 1-point increase on seven-point scale, $\mathrm{P}<0.001)$ were both independently associated with sometimes or often de-escalating PPIs (Table 2). Gender and years in practice had no effects. The predicted probability of de-escalating PPI for respondents reporting the highest level of concern about PPI harms ( 7 on the $1-7$ scale), with other covariates at their means, was $96 \%$ versus $71 \%$ for a concern level at the midpoint of the scale (4), and $20 \%$ for the lowest level of concern (1). As a subgroup analysis, we evaluated the effects of attending physicians' practice characteristics (practice setting, specialization, academic affiliation, and patient volume) on changes in prescribing habits. None were significantly associated with PPI de-escalation.

\section{Discussion}

PPIs have been linked with a growing list of serious adverse effects, but the clinical significance of these findings continues to be debated. The magnitude of the excess risk for most of the adverse effects is small, and it remains unclear whether PPIs are the cause of these adverse effects, or are linked to them in a non-causal way $[14,26]$. Against the backdrop of this uncertainty, little is known about physicians' perceptions of possible adverse effects and what effect these concerns are having on prescribing behavior. In this survey, we found that internists in the United States are universally familiar with one or more reported PPI adverse effects and are concerned about them. Furthermore, prescribers are changing their PPI prescribing habits despite the ongoing debate about the true risks PPIs pose. Nearly three-quarters of respondents sometimes or often take steps to de-escalate PPIs, and physicians with greater familiarity and concern are more likely to do so. These findings clearly demonstrate that evidence of possible PPI adverse effects is creating a measure of alarm among physicians.

Respondents reported using several different strategies to de-escalate PPIs. They most frequently reduced the PPI dose, followed by switching PPI to an $\mathrm{H} 2$ blocker, and simply discontinuing PPIs. All of these strategies have been used in PPI de-escalation studies, but none is clearly favored based on available evidence. One RCT compared tapering doses of PPI versus abrupt withdrawal and found no difference in symptom control at 12 months [27]. Other investigators have examined the efficacy of either step-down therapy [20] or abrupt discontinuation [19] in non-randomized studies, but did not compare them head-to-head. The step-down and $\mathrm{H} 2$ block substitution strategies have theoretical appeal given the possibility of physiologic acid rebound hyper-secretion after PPI withdrawal, although the clinical significance of this phenomenon remains unclear [28].

Understanding when and how physicians make decisions 
Table 1. Demographic and practice Characteristics of Respondents $(\mathrm{N}=487)$

\begin{tabular}{|c|c|}
\hline Characteristic & n $(\%)$ \\
\hline \multicolumn{2}{|l|}{ Age } \\
\hline$<40$ & $182(37.4 \%)$ \\
\hline $40-59$ & $225(46.2 \%)$ \\
\hline$>59$ & $80(16.4 \%)$ \\
\hline \multicolumn{2}{|l|}{ Sex } \\
\hline Male & $343(70.4 \%)$ \\
\hline Female & $144(29.6 \%)$ \\
\hline \multicolumn{2}{|l|}{ Training level } \\
\hline Residency & $80(16.4 \%)$ \\
\hline Fellowship & $26(5.3 \%)$ \\
\hline Attending & $381(78.2 \%)$ \\
\hline \multicolumn{2}{|l|}{ Specialty $^{\#}$} \\
\hline Internal medicine (general medicine or hospitalist) & $282(74.0 \%)$ \\
\hline Gastroenterology & $11(2.9 \%)$ \\
\hline Other medical subspecialist & $88(23.1 \%)$ \\
\hline \multicolumn{2}{|l|}{ Board certification $^{\#}$} \\
\hline No & $10(2.6 \%)$ \\
\hline Yes & $371(97.4 \%)$ \\
\hline \multicolumn{2}{|l|}{ Years in practice ${ }^{\#}$} \\
\hline$<5$ years & $43(11.3 \%)$ \\
\hline $5-10$ & $71(18.7 \%)$ \\
\hline $11-15$ & $46(12.1 \%)$ \\
\hline $16-20$ & $53(14.0 \%)$ \\
\hline$>20$ & $167(44.0 \%)$ \\
\hline \multicolumn{2}{|l|}{ Patients seen per week ${ }^{\#}$} \\
\hline$<26$ & $31(8.2 \%)$ \\
\hline $26-50$ & $107(28.2 \%)$ \\
\hline $51-75$ & $108(28.4 \%)$ \\
\hline $76-100$ & $83(21.8 \%)$ \\
\hline$>100$ & $51(13.4 \%)$ \\
\hline \multicolumn{2}{|l|}{ Practice setting ${ }^{\#}$} \\
\hline All outpatient & $137(36.1 \%)$ \\
\hline Primarily outpatient & $140(36.8 \%)$ \\
\hline All inpatient & $63(16.6 \%)$ \\
\hline Primarily inpatient & $40(10.5 \%)$ \\
\hline \multicolumn{2}{|l|}{ Medical school affiliation ${ }^{\#}$} \\
\hline No & $195(51.3 \%)$ \\
\hline Yes & $185(48.7 \%)$ \\
\hline
\end{tabular}

\#Attending physicians only. 


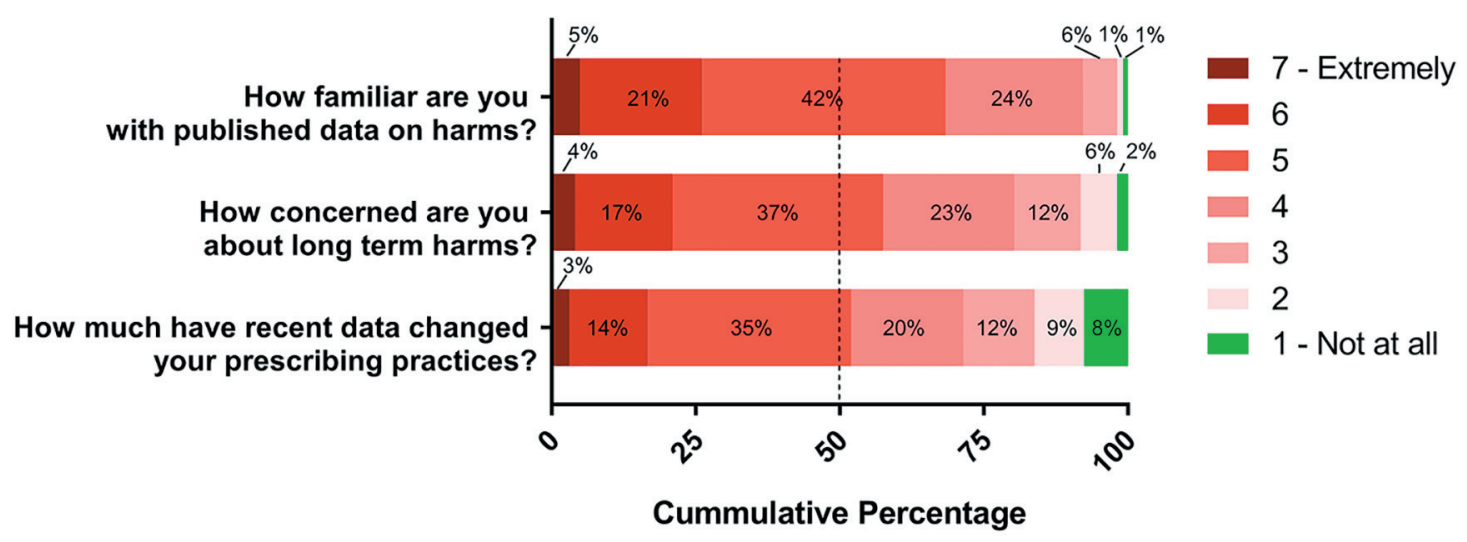

Figure 1. Respondents' familiarity with and concern about PPI harms, and changes in prescribing practices on seven-point scale. Dashed line represents median score.

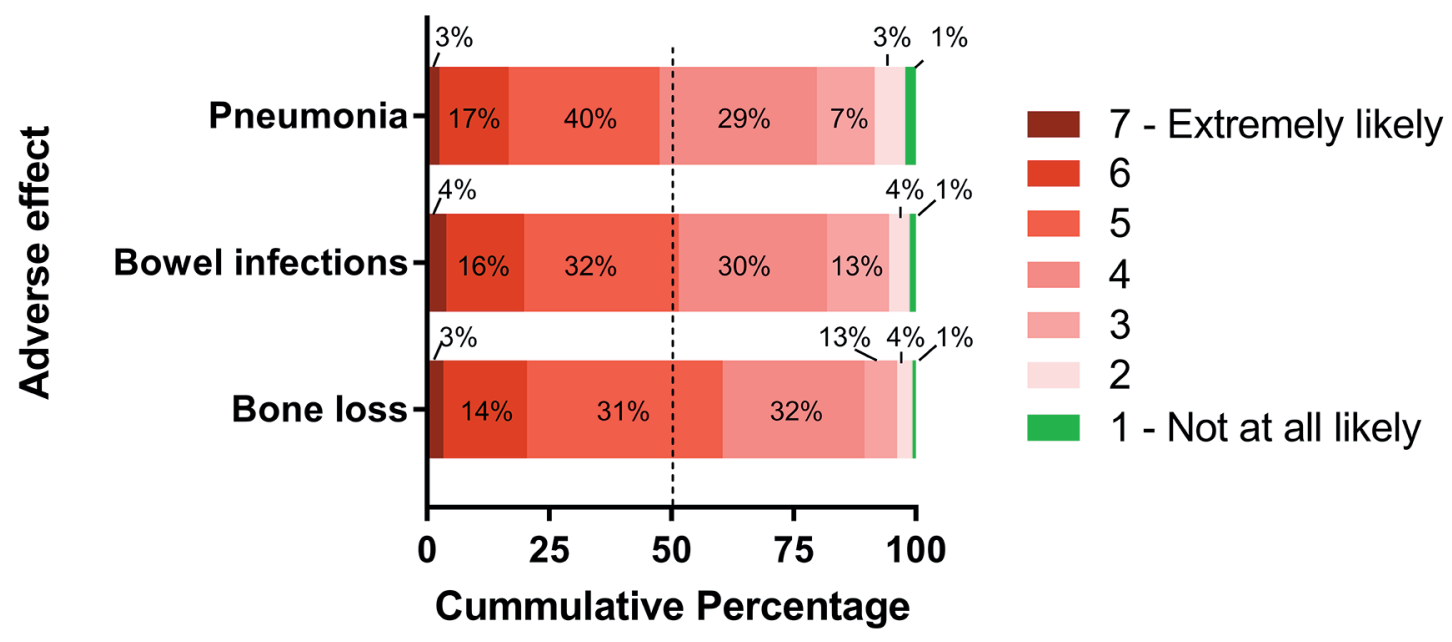

Figure 2. Respondents' perceived likelihood that long-term PPI use increases the risk of specific adverse effects on seven-point scale. Dashed line represents median score.

about PPI de-escalation is critical to ensuring that patients who truly need effective anti-secretory therapy continue to receive it and those patients who do not are taken off such medications. All PPI de-escalation strategies are not equally appropriate for all patients. For patients who require prophylactic antisecretory therapy for prevention of upper gastrointestinal (GI) bleeding, evidence suggests that $\mathrm{H} 2$ blockers provide only a fraction of the protection that PPIs do [22, 29, 30]. Therefore, in such patients, switching PPI to an H2 blocker would be an inappropriate strategy. On the other hand, for patients with un- complicated gastroesophageal reflux disease (GERD), ongoing anti-secretory therapy may not be necessary at all [31].

Physicians must first make individualized risk-benefit decisions about the necessity of a PPI, and then decide the best de-escalation strategy if a PPI is not needed. However, it appears that these decisions are often discordant with best evidence. In an analysis of different data from the same survey, we found that when concerned about the possibility of an adverse effect, such as osteoporosis, physicians are more likely to recommend stopping PPIs in patients who use them

Table 2. Association of Physician Characteristics With Sometimes or Often Reducing PPI in Logistic Regression Model

\begin{tabular}{|c|c|c|c|c|}
\hline Variable & Unadjusted OR (95\% CI) & P-value & Adjusted OR (95\% CI) & P-value \\
\hline Female & $1.56(0.98-2.49)$ & 0.06 & $1.67(0.98-2.83)$ & 0.06 \\
\hline Years in practice (per decade) & $0.99(0.90-1.10)$ & 0.90 & $1.06(0.94-1.19)$ & 0.33 \\
\hline Concern about PPI harms (1-point change on seven-point scale) & $2.26(1.87-2.74)$ & $<0.001$ & $2.14(1.76-2.61)$ & $<0.001$ \\
\hline
\end{tabular}


for the prevention of aspirin-related upper GI bleeding versus GERD [21]. This surprising finding may represent a greater willingness on the part of physicians to continue potentially harmful medications when used for the treatment of symptoms compared to when used for preventive purposes. Physicians may benefit from interventions to help them appropriately individualize anti-secretory therapy.

Our findings raise a number of questions that merit future investigation. In real-world practice, how frequently are physicians actually taking steps to reduce PPI exposure, amid the myriad other demands during clinic visits? Which, if any, patient populations are they targeting for reduction of PPI exposure? Are patients whose PPIs are dose-reduced eventually given a trial off PPI entirely? For the most significant looming question of all - the true relationship between PPIs and reported adverse effects - an RCT may eventually be required.

Our study has a number of strengths and weaknesses. The survey included a nationally representative sample of internists in the United States and had a high response rate, suggesting that our results are generalizable. However, the survey was carried out in 2013, when studies linking PPIs to several additional adverse effects, including chronic kidney disease, dementia, and stroke, had not yet been published. These newer studies would likely further heighten prescribers' concerns about PPIs. As with any survey of medical decision making, decisions endorsed by respondents may reflect social desirability bias.

In conclusion, we have found that US internists report high levels of awareness and concern about PPI adverse effects. Furthermore, they are changing their prescribing habits to de-escalate PPIs in response. Physicians are likely to benefit from specific guidance about which of their patients can safely stop PPIs, and about which of many strategies for de-escalation are most likely to succeed.

\section{Grant Support}

This project was supported by the University of Michigan. The contents do not represent the views of the US Department of Veterans Affairs or the US Government.

\section{Conflict of Interest}

Jacob E. Kurlander has received research funding from Ironwood Pharmaceuticals; Mark Kolbe: none; Joel H. Rubenstein: none; Caroline Richardson: none; Michele Heisler: none; Arlene Weissman: none; Raymond De Vries: none; Sameer D. Saini has served as consultant for FMS, Inc.

\section{Author Contributions}

Jacob E. Kurlander: analysis and interpretation of data; drafting of the manuscript; critical revision of the manuscript for important intellectual content; Mark Kolbe: study concept and design; critical revision of the manuscript for important intellectual content; Joel H. Rubenstein: analysis and interpreta- tion of data; critical revision of the manuscript for important intellectual content; Caroline Richardson: analysis and interpretation, critical revision of the manuscript for important intellectual content; Michele Heisler: analysis and interpretation, critical revision of the manuscript for important intellectual content; Arlene Weissman: study concept and design; acquisition of data; Raymond De Vries: analysis and interpretation of data; critical revision of the manuscript for important intellectual content; Sameer D. Saini: study concept and design; analysis and interpretation of data; critical revision of the manuscript for important intellectual content.

\section{References}

1. Most Prescribed Drugs in the U.S. http://drugs.healthgrove.com/stories/5221/25-most-prescribed-drugs-inthe-u-s. Accessed October 10, 2016.

2. Atkins AM, Sekar RMC. Proton pump inhibitors: Their misuse, overuse and abuse. IOSR J Pharm. 2013;3(2):2529.

3. Metaxas ES, Bain KT. Review of proton pump inhibitor overuse in the US Veteran population. J Pharm Technol. 2015;31(4):167-176.

4. Vaezi MF, Yang YX, Howden CW. Complications of Proton Pump Inhibitor Therapy. Gastroenterology. 2017;153(1):35-48.

5. Sehested TS, Fosbøl EL, Hansen PW, Charlot MG, TorpPedersen C, Gislason GH. Proton pump inhibitor use increases the associated risk of first-time ischemic stroke. A Nationwide Cohort Study. Circulation. 2016;134(Suppl 1):A18462-A18462.

6. Gomm W, von Holt K, Thome F, Broich K, Maier W, Fink A, Doblhammer G, et al. Association of proton pump inhibitors with risk of dementia: a pharmacoepidemiological claims data analysis. JAMA Neurol. 2016;73(4):410416.

7. Lambert AA, Lam JO, Paik JJ, Ugarte-Gil C, Drummond MB, Crowell TA. Risk of community-acquired pneumonia with outpatient proton-pump inhibitor therapy: a systematic review and meta-analysis. PLoS One. 2015;10(6):e0128004.

8. Tran-Duy A, Spaetgens B, Hoes AW, de Wit NJ, Stehouwer CD. Use of proton pump inhibitors and risks of fundic gland polyps and gastric cancer: systematic review and meta-analysis. Clin Gastroenterol Hepatol. 2016;14(12):1706-1719 e1705.

9. Zhou B, Huang Y, Li H, Sun W, Liu J. Proton-pump inhibitors and risk of fractures: an update meta-analysis. Osteoporos Int. 2016;27(1):339-347.

10. Janarthanan S, Ditah I, Adler DG, Ehrinpreis MN. Clostridium difficile-associated diarrhea and proton pump inhibitor therapy: a meta-analysis. Am J Gastroenterol. 2012;107(7):1001-1010.

11. Lazarus B, Chen Y, Wilson FP, Sang Y, Chang AR, Coresh J, Grams ME. Proton pump inhibitor use and the risk of chronic kidney disease. JAMA Intern Med. 2016;176(2):238-246.

12. Shah NH, LePendu P, Bauer-Mehren A, Ghebremariam 
YT, Iyer SV, Marcus J, Nead KT, et al. Proton pump inhibitor usage and the risk of myocardial infarction in the general population. PLoS One. 2015;10(6):e0124653.

13. Laine L, Nagar A. Long-term PPI use: balancing potential harms and documented benefits. Am J Gastroenterol. 2016;111(7):913-915.

14. Freedberg DE, Kim LS, Yang YX. The risks and benefits of long-term use of proton pump inhibitors: expert review and best practice advice from the American Gastroenterological Association. Gastroenterology. 2017;152(4):706715 .

15. Morris ZS, Wooding S, Grant J. The answer is 17 years, what is the question: understanding time lags in translational research. J R Soc Med. 2011;104(12):510-520.

16. Davis D, Evans M, Jadad A, Perrier L, Rath D, Ryan D, Sibbald G, et al. The case for knowledge translation: shortening the journey from evidence to effect. BMJ. 2003;327(7405):33-35.

17. Span P. Study finds growing reason to be wary of some reflux drugs. The New York Times. http://www.nytimes. com/2016/02/02/health/study-finds-growing-reason-tobe-wary-of-some-reflux-drugs.html. Published January 29, 2016. Accessed January 7, 2017.

18. Lee BY. How safe are heartburn medications? Forbes. http://www.forbes.com/sites/brucelee/2016/01/12/howsafe-are-heartburn-medications-and-are-they-overused/. Accessed January 7, 2017.

19. Reimer C, Bytzer P. Discontinuation of long-term proton pump inhibitor therapy in primary care patients: a randomized placebo-controlled trial in patients with symptom relapse. Eur J Gastroenterol Hepatol. 2010;22(10):11821188.

20. Inadomi JM, Jamal R, Murata GH, Hoffman RM, Lavezo LA, Vigil JM, Swanson KM, et al. Step-down management of gastroesophageal reflux disease. Gastroenterology. 2001;121(5):1095-1100.

21. Kurlander JE, Kolbe M, Scheiman JM, Weissman A, Piette JD, Rubenstein JH, Waljee AK, et al. The right idea for the wrong patient: results of a national survey on stopping PPIs. Clin Gastroenterol Hepatol. 2017;15(9):1475-1476.

22. Mo C, Sun G, Wang YZ, Lu ML, Yang YS. PPI versus
Histamine $\mathrm{H} 2$ receptor antagonists for prevention of upper gastrointestinal injury associated with low-dose aspirin: systematic review and meta-analysis. PLoS One. 2015;10(7):e0131558.

23. Internal medicine insider research panel. https://iminsider.org/. Accessed November 14, 2016.

24. VanKim NA, Erickson DJ, Laska MN. Food shopping profiles and their association with dietary patterns: a latent class analysis. J Acad Nutr Diet. 2015;115(7):11091116.

25. Wynia MK, VanGeest JB, Cummins DS, Wilson IB. Do physicians not offer useful services because of coverage restrictions? Health Aff (Millwood). 2003;22(4):190-197.

26. Schoenfeld AJ, Grady D. Adverse effects associated with proton pump inhibitors. JAMA Intern Med. 2016;176(2):172-174.

27. Bjornsson E, Abrahamsson H, Simren M, Mattsson N, Jensen C, Agerforz P, Kilander A. Discontinuation of proton pump inhibitors in patients on long-term therapy: a double-blind, placebo-controlled trial. Aliment Pharmacol Ther. 2006;24(6):945-954

28. Lodrup AB, Reimer C, Bytzer P. Systematic review: symptoms of rebound acid hypersecretion following proton pump inhibitor treatment. Scand J Gastroenterol. 2013;48(5):515-522.

29. Lanas A, Garcia-Rodriguez LA, Arroyo MT, Bujanda L, Gomollon F, Forne M, Aleman S, et al. Effect of antisecretory drugs and nitrates on the risk of ulcer bleeding associated with nonsteroidal anti-inflammatory drugs, antiplatelet agents, and anticoagulants. Am J Gastroenterol. 2007;102(3):507-515.

30. Prevention of NSAID-related ulcer complications. American College of Gastroenterology. http:/gi.org/guideline/ prevention-of-nsaid-related-ulcer-complications/. Accessed December 27, 2016.

31. Haastrup PF, Paulsen MS, Christensen RD, Sondergaard J, Hansen JM, Jarbol DE. Medical and non-medical predictors of initiating long-term use of proton pump inhibitors: a nationwide cohort study of first-time users during a 10-year period. Aliment Pharmacol Ther. 2016;44(1):7887. 